Coping with variability of location sensing in large-scale ubicomp environments Peer-reviewed author version

AKSENOV, Petr; LUYTEN, Kris \& CONINX, Karin (2009) Coping with variability of location sensing in large-scale ubicomp environments. In: International Workshop on Sensing and Acting in Ubiquitous Environments (SEACUBE'09)..

Handle: http://hdl.handle.net/1942/10351 


\title{
Coping with variability of location sensing in large- scale ubicomp environments
}

\author{
Petr Aksenov, Kris Luyten, and Karin Coninx \\ Hasselt University - transnationale Universiteit Limburg \\ Expertise centre for Digital Media - IBBT \\ Wetenschapspark 2, BE-3590 Diepenbeek, Belgium \\ \{petr.aksenov, kris.luyten, karin.coninx\}@uhasselt.be
}

\begin{abstract}
The work addresses the problem of coping with a diversity of location tracking techniques available in ubiquitous computing environments. We investigate how this diversity can be embedded in the environment in a way that typical difficulties coming from using location-awareness are hidden. We present an approach to improve location-awareness of these environments by means of integrating the knowledge about different location systems into an existing framework for designing pervasive environments in the form of an ontology. Emerging challenges are also discussed in the context of continuous and smooth communication.
\end{abstract}

Keywords- large-scale environments, location systems, locationawareness, pervasive applications, spatial ontology

\section{INTRODUCTION}

Since the first context-aware applications, location has always been a central aspect in determination of the context of use of an application and is the subject of many research efforts. We can roughly subdivide this research into three areas: (1) research into the location determination itself which is mainly focused on the hardware, (2) research into creating easy access to location data and their further processing in accordance with the tasks they are used in, and (3) research into the specific class of applications that we label "location-aware". Examples of (1) can be found elsewhere, e.g. Active Bat [1] or IRIS-LPS, an optical infrared local positioning system developed for local use [2], and they describe new approaches and techniques to determine location, whereas (3) is focused on what type of applications can be improved by using locationawareness. In this paper we deal with a combination of (2) and (3) and investigate how the diversity in location tracking can be embedded in large scale ubicomp environments in such a way that typical difficulties coming from using location-awareness are hidden from both the developer and the user. Getting location data that, according to the application needs, is accurate enough is mostly a cumbersome task. Additionally, as it has been pointed out in a great deal of publications on the topic (for example, [3]), referring to spatial information in the qualitative, approximate way is natural to a person, with the quantitative way left to machines. And the qualitative way is

Part of the research at EDM is funded by EFRO (European Fund for Regional Development) and the Flemish Government. Funding for this research was also provided by the Research Foundation - Flanders (F.W.O. Vlaanderen, project CoLaSUE, number G.0439.08N.).

9781-4244-3941-6/09/\$25.00 @2009 IEEE never precise. Interpretation of expressions like "on the right", "in front of", "not far from" always depends on the situation and, for example, "on the right" may mean a certain area of space rather than a direction. Also, in some situations the knowledge available can simply be insufficient for making an exact judgement. These two factors together bring uncertainty into the problem of dealing with location and this issue must be handled somehow. Since devices have become a crucial part of our life, it is natural to request them to behave and act in such a way that we, as users, would pay as least attention and effort to processing the information we receive as possible. As Weiser stated it, "...to make a computer so imbedded, so fitting, so natural, that we use it without even thinking about it..." [4]. An application is powerful when it is sustainable to changes so that, in the case of a change, it preserves its functionality. However, the difficulty of this task is proportional to the complexity of the environment. When it goes to large-scale, delays are inevitable due to the increased amount of relations between resources and thus information to be communicated. In a pervasive environment, it becomes even more demanding due to the high mobility of interacting resources: a change must happen as quickly and be unnoticeable to the users as possible; otherwise it affects the satisfaction of the user. From the spatial point of view, an environment can be considered sustainable if changes in location sensing are handled accordingly and appropriate corrections are made to the system in a harmless way.

In our previous work we have introduced a new usercentred approach to represent spatial information in a humanfriendly way with the ability to address incomplete and uncertain spatial knowledge. We called that approach the Ambient Compass [5] which we briefly overview in section IV.A. The current work describes an extension to our Ambient Compass and addresses the question of adapting spatial information processing to the general case of any location system by means of making this system a part of the approach.

\section{RELATED WORK}

A very well known survey of location systems available for ubiquitous computing was presented by [6] yet in 2001. Similar discussions still take place nowadays [8], and looking at the two, one can find just a couple of techniques mentioned in both. This means that new systems for location tracking appear 
and better meet the needs of nowadays environments and technological progress. Today, there is a plethora of positioning systems available, both commercial and from academia, which vary in a whole bunch of characteristics: provided accuracy, cost, installation requirements, equipment burdens, the ease of use, and so on. Some of these systems, such as GPS, have been on stage for many a year and proved to be an acceptable and sufficient solution for location in certain types of tasks. For example, the above GPS technology can be nowadays found as the dominant location provider in the majority of the outdoor tasks. However, indoors or in heavily loaded urban city environments, the requirements on the capabilities and output from the location system involved are stricter and more demanding, thus making the situation not that trivial.

Several location systems can co-exist in the same environment. The problem of using more than one location system in the environment is not novel, and several viewpoints on dealing with it can be distinguished. At first, in many cases the efforts are concentrated on the so called "sensor fusion" approach [6] in which the output data of every location system are used together, usually to get a better accuracy. For example, Hii and Zaslavsky [9] report an improved accuracy that is a result of combining a Wi-Fi based real time location system with an acoustic location system taking an advantage of that two very different techniques complement to each other. Leonhardt and Magee [10] also combine several location systems into an acquisition function to get the final location. The main interest of this work to us is, however, in that they propose an idea of location service as a completely separate functionality with an open and scalable architecture so that new sensing technologies can be integrated. In this regard, Middlewhere [11] is another example of approaching the support of location information as an independent service separated from the rest of the application. Additionally, the authors explicitly focus on providing support and integration of new location systems at run-time, the property so natural and important in today's heterogeneous environments. Next, the problem of combining indoor and outdoor situations has got a special separate interest due to the particular change in the technologies used for sensing. For example, Mok et al. [12] reported the results of combining a GNSS outdoor technology with a Wi-Fi based indoor technology, and, among the rest, studied the signal strength of each of these technologies and the way it affects the determined location around the indooroutdoor transition areas. In the work by Hansen et al. [13], four different algorithms of switching between GPS and a Wi-Fi technology were performed in a situation involving both outdoor and indoor areas. Despite the work says nothing about the techniques used to provide a handover from one location system to the other, the results of the experiments clearly indicated the need for such kind of symbiosis. Another interesting example of multiple location systems tied together is given by Coronato and Esposito [14] where the authors combine a RFID and a Wi-Fi-based location system to address the problem of locating doctors and patients in a hospital. They also provide location-aware support to doctors in terms of displaying patient's details on a wall monitor. A special interest here is in that a location system is considered as part of an ontology of physical locations, which allows reasoning and resolving location conflicts, if such appear. The focus of the work, however, is put on combining measurements from both systems in order to choose the more trusted one, and not on a possible handover, therefore the location systems' further infrastructure is not considered.

As these and many other works in the field show, providing a universal solution for location determination is still an open problem since available location tracking technologies are highly influenced by the diversity of infrastructures and tasks executed in the environment. As a result, their application is successful only in limited areas. A possible step towards bridging this gap is to consider multiple location systems in combination with each other and we have provided several examples of related work on such attempts. Different to this existing work, we consider the very process of switching between location systems at run-time by means of making the supporting framework aware of the details of the involved location systems while still keeping it independent from the particular tracking systems.

\section{LARGE SCALE UBICOMP APPLICATIONS IN REWIRE}

ReWiRe [15] is a component-based framework for designing interactive pervasive computing environments. It is a system that adapts itself when new configurations such as the usage of a new mobile device arise. The approach used in ReWiRe links the environment configuration with the software architecture (a set of connected components) and this link is maintained continuously during the lifetime of a pervasive application. When the environment configuration changes, the software architecture changes accordingly. For this purpose a pervasive system is encoded as a graph structure that describes how entities in the environment relate to each other. A graph corresponds to an instance of an OWL-ontology (http://www.w3.org/2004/OWL/) which includes concepts to represent generic resources found in a pervasive computing environment such as users, computing devices, services, tasks, etc., and allows querying, updating and rewiring the system while it is being used. The component-based architecture of ReWiRe extended with the Ambient Compass can cope with spatial changes of resources just as it handles other changes in the environment. By further allowing it to react on changes in the spatial relationships, we empower applications built with ReWiRe with the ability to reason on the quality of location tracking at the same time with ordinary location changes.

\section{HANDLING SPATIAL INFORMATION}

Various techniques to obtain information about location of an object from sensors exist [6], [7], [16], [17], [18]. Considerable effort is constantly put on improving the quality of measured location: the technologies behind existing location systems are improving, new ones appear, and it is quite certain that this development continues and will result in more accurate and robust location-sensing technologies. But in every case, the sensed data is used for updating the existing spatial model of the environment and for deriving spatial relations between resources. And if a smooth transition between different location systems within the same environment is possible, user's frustration from, and confusion about, being confronted with varying degrees of precision can be minimised. 

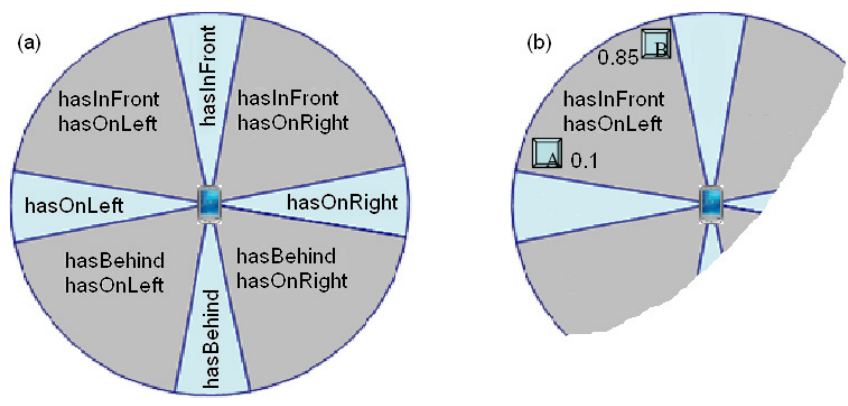

Figure 1. (a) The Ambient Compass divides the space around a resource into eight zones; (b) resources belonging to the same zone of the compass are distinguished by means of assigning each of them a degree of membership to this zone.

\section{A. Ambient Compass}

Aggregated with the upper ReWiRe ontology, there is an ontology of spatial concepts. This spatial ontology provides a structured description of the spatial context of resources in the environment, which can be exploited, for instance, to improve a distribution algorithm for user interfaces amongst multiple screens as well as to improve location-awareness of users in (unfamiliar) computer-augmented environments.

Spatial relationships are determined by the Ambient Compass (Fig. 1). The compass defines several basic concepts typical to spatial structures, such as position, orientation and four directions - right, left, front, and behind - and is resourcecentred, meaning that every resource sees other resources through its own compass. The proposed classification aims at giving an application the possibility to speak a language similar to that of humans when they talk about spatial arrangements.

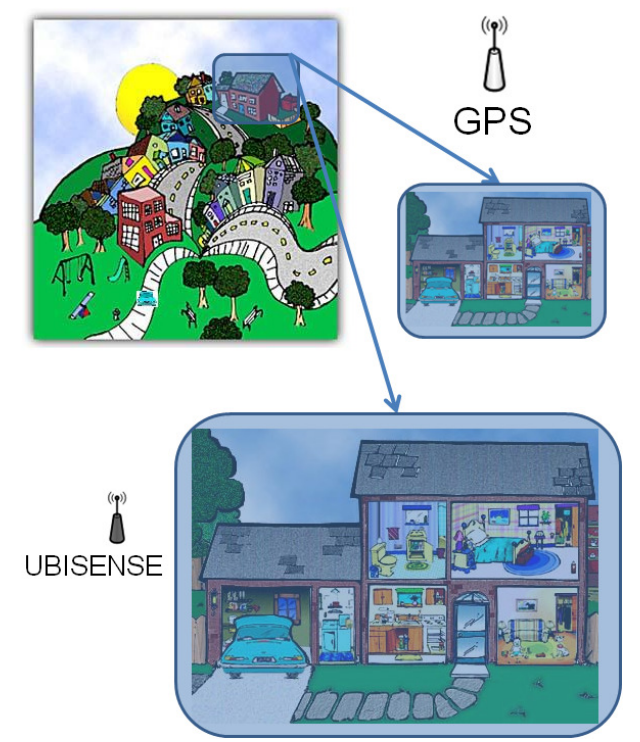

Figure 2. The outdoor area and the building are controlled by different location systems and different type of location data is sent to the application in each case. The creation of the figure was inspired by and is based on the images seen on the website of the U.S. Environmental Protection Agency (http://www.epa.gov/kidshometour/).
In general, two more cardinal directions, "below" and "above", can be identified but due to the complexity that comes with adding one more dimension and since a planar description already covers a wide range of applications, we did not include them in the current representation. Furthermore, people's perception of objects in the "above/below" directions turns out to be greatly asymmetric as opposed to, for example, the "left/right" pair [19]. Therefore it requires further study towards a modified approach in order to gain better efficiency. Also, time plays an important role in our consideration, and in our previous work on spatial relations [20] we proposed an idea to predict the trajectory of a moving resource based on its prior locomotion and future intention. The use of the Ambient Compass through the timeline to support this kind of tasks can be beneficial.

\section{B. Integrating location systems with ReWiRe}

In Fig. 2 the user outdoors (top-left) is tracked by GPS and finds the way to the building successfully. Having walked inside, the user decides to use a wall-mounted large screen to see the photos he has just taken with his smartphone, and kept being located by GPS (top-right), is given a false negative solution because his detected location is 5 metres away from the actual one and is in the adjacent room. If located by Ubisense, which is installed in the building (bottom), the determined solution would be correct since the accuracy of the Ubisense set up in that building is around 0.5 metres. But in order to make the user aware of a higher level of trust in the measured accuracy, knowing the currently active location system's details is important.

Formerly a location system was considered in our framework only externally as a means to provide the necessary location data. The Ambient Compass itself did not take into account the quality of location measurements. However, in this case it is not clear how the application should adapt its calculations when location tracking is taken over by another location system and the layout of the compass should be recalculated. Without knowing the corresponding characteristics of the location system currently in use, such a transition between two compass states may be impossible or can lead to erroneous and unsuitable results; whereas awareness of this type of change lets the application adjust the layout of the compass and thus connect interacting resources spatially correctly. For example, Fig. 3 shows a situation when the same environment is tracked by two different location systems, resulting in two different sets of spatial relations calculated for the same arrangement of interacting resources in the environment. Therefore incorporating the knowledge about location systems into the framework can be beneficial.

Like in the case of the spatial ontology, a location system is described in a separate ontology which is built-over the upper environment ontology in ReWiRe. It provides basic data about the location system, such as its update rate, standard error, availability. Knowing these details brings the benefit of correct on-the-fly spatial support in a larger number of areas and situations. Additionally, this type of support opens other areas of improving the intelligence and broadening the functionality of the environment. For example, the already recalled idea of predicting a possible future trajectory of a moving resource to a 


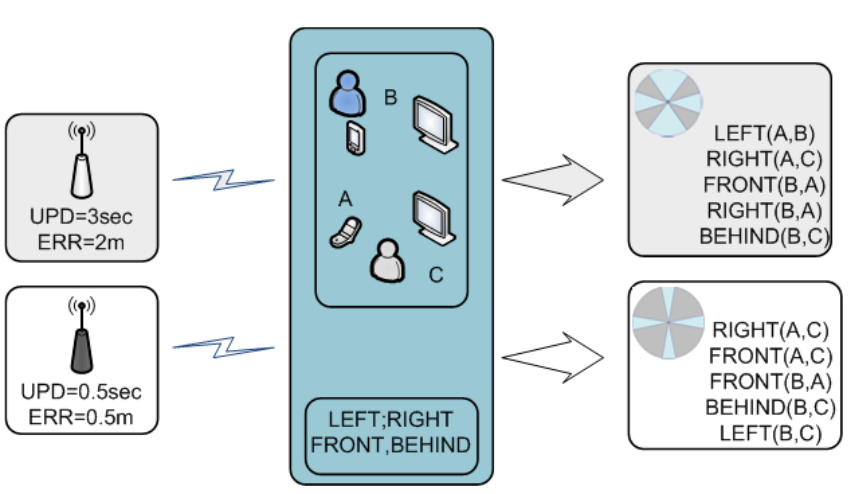

Figure 3. Different location systems have different characteristics. Based on these characteristics, the layout of the ambient compass is defined and spatial relationships are calculated as appropriate.

large extent depends on how reliable the positioning through the timeline is.

\section{ONGOING DEVELOPMENT}

Currently we are working on the structure of the location system's ontology and its further integration into ReWiRe. For validation, we use Ubisense, a precise real-time location system that provides very accurate location tracking of people and assets [7]. It consists of a set of firmly fixed Ubisensors that track locations of Ubitags throughout a certain area defined by the position and orientation of the sensors. Our installation of the "Ubisense Research Package" comprising four sensors and ten tags is done in an area of approximately $3.5 \times 3.5$ metres. The Ubisense system has a possibility to change the update rate of its location measurements. In our terms, this feature creates a different location system and will be used to simulate a change of the currently active location system, thereby serving as a basis for verification of the approach.

As a test scenario, we consider a user with a PDA who is oriented towards the left display and is projecting something from the PDA on it. The right display is inactive which is indicated by a dashed line (Fig. 4). At a certain moment, the user begins to turn clockwise so that the right display moves from the PDA's perspective - from being equally "isInFrontOf" and "isOnRightOf" to much more "isInFrontOf"; whereas the left display starts holding both "isOnLeftOf" and "isInFrontOf" relations. When the PDA's rotation reaches a certain angle, the image of the PDA is copied to the right display, activating it (the dashed line becomes solid), but still being shown on the left one as well. Having observed that the PDA keeps turning, the compass discovers that the left display, though still staying in front, is already considerably to the left of the PDA and therefore can be released (the solid line becomes dashed).Preliminary tests of the Ubisense installation revealed that this original scenario could not be completed because the location accuracy was insufficient for introducing orientation of a device of the size of a PDA. Therefore the scenario was replaced with that of the PDA moving along three displays, as shown in Fig. 5, with the same intention of implementing location-aware switching between the screens as the PDA moves.
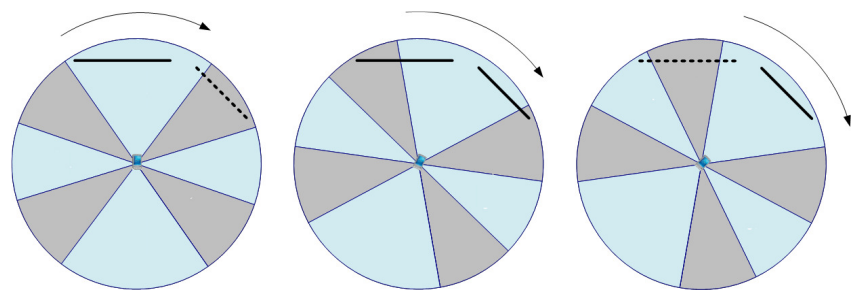

Figure 4. The displays change their status from inactive (dashed line) into active (solid line), and vice versa, in response to the PDA turning clockwise. The change of the active display is preceded by the state when the image is shown on both of them.

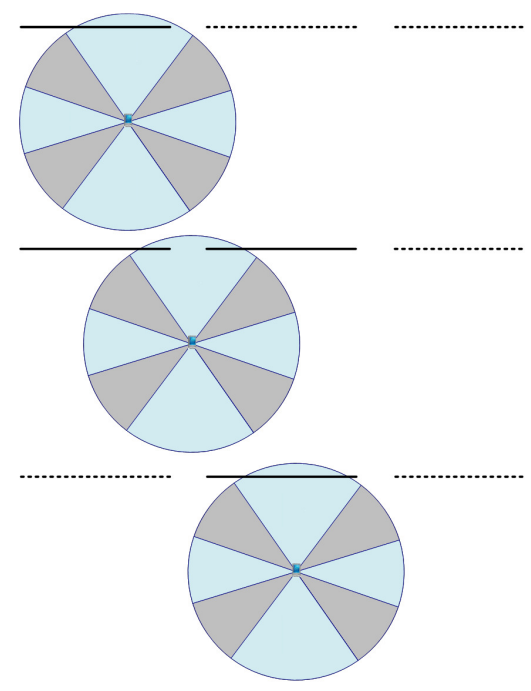

Figure 5. As the PDA oriented towards the displays moves from left to right, the state of these displays changes accordingly.

\section{DISCUSSION}

Different collections of technologies can be identified in different connected places, certainly in large-scale ubicomp spaces. People might move from one place to another to find a different set of technologies that can assist in the same type of tasks. Location tracking is among the technologies that are gaining importance, specifically on a large-scale. However, it is unlikely that the same system is installed everywhere since the requirements, infrastructure and the needs for location data vary greatly. Even identical technologies can produce different output depending on such factors as, for example, the requirements and capabilities of the environment and implementation. Nevertheless, smooth and seamless communication of resources interacting throughout and between these environments is a crucial attribute, and here we see two items to be brought to the discussion.

At first, in many situations, investigation and application of a location-tracking technology is confined to a single task (or a small group of tasks) in a single place, thus leaving the question of global overall applicability of the proposed technique out of the consideration. Besides, to the best of our knowledge, immediate support of an arbitrary localisation technology at run-time still remains an important step that 
needs to be taken for large-scale ubicomp environments. In the presented work, we have described our ongoing attempt to combine an arbitrary task and an arbitrary location system into one problem since we believe that with the emergence of new location systems and upgrades of the existing ones such kind of support will come into the leading role in pervasive environments.

Together with the outlined approach we face the second challenge for a characteristic a successful pervasive application must possess - the continuity of the user interface responsible for the communication between the application and the user. From a perspective of dealing with location systems in a complex environment, switching between them as is may be error-prone. This, in turn, may cause unwanted delays or even gaps and disconnects in the interaction process between the application and the user. Since in large-scale environments user interfaces responsible for that interaction process are often distributed, the presence of such flaws will contradict with the important requirement of continuity of (distributed) user interfaces [21]. Our approach to improve location-awareness in large scale ubicomp environments aids, in particular, in preserving continuity of the associated user interfaces of applications that are deployed in such an environment by means of making transition between location systems more reliable.

\section{REFERENCES}

[1] A. Harter, A. Hopper, P. Steggles, A. Ward, and P. Webster, "The anatomy of a context-aware application", in Proc. of the $5^{\text {th }}$ ACM/IEEE Int. Conf. on Mobile Computing and Networking, MOBICOM'99, Seattle, WA, USA, Aug 1999, pp. 59-68.

[2] E. Aitenbichler and M. Mühlhäuser, "An IR local positioning system for smart items and devices", in Proc. of the $3^{\text {rd }}$ Int. Workshop on Smart Appliances and Wearable Computing, IEEE Computer Society Press, Los Alamitos, CA, USA, 2003, pp.334-339.

[3] B. Lorenz and H.J. Olhbach, "A hybrid model for indoor spatial reasoning", in Proc. $1^{\text {st }}$ Int. Workshop on Mobile Geospatial Augmented Reality, Banff, Alberta, Canada, May 2006.

[4] M. Weiser, "Ubiquitous computing" [Online]. Available: http://www.ubiq.com/hypertext/weiser/UbiHome.html

[5] P. Aksenov, G. Vanderhulst, K. Luyten, and K. Coninx, "Ambient Compass: One approach to model spatial relations", in Proc. of $13^{\text {th }}$ Int. Conf. on Human-Computer Interaction, HCII 2009, LNCS, vol. 5620, pp.183-191.
[6] J. Hightower and G. Boriello, "Location systems for ubiquitous computing", Computer, vol. 34, no. 8, pp. 57-66, Aug 2001.

[7] Ubisense, http://www.ubisense.net.

[8] R. Mautz, "Combination of indoor and outdoor positioning", in Proc of $I^{\text {st }}$ Int. Conf. on Machine Control and Guidance, June 2008, pp. 79-88.

[9] P. Hii and A. Zaslavsky, “ Improving location accuracy by combining WLAN positioning and sensor technology", in Proc. of $1^{\text {st }}$ Workshop on Real-World Wireless Sensor Networks, REALWSN, Stockholm, 2005

[10] U. Leonhardt and J. Magee, "Multi-sensor location tracking", in Proc. of $4^{\text {th }}$ Ann. ACM/IEEE Conf. on Mobile Computing and Networking, MOBICOM '98, Dallas, Texas, USA, Oct 1998, pp. 203-214.

[11] A. Ranganathan, J. Al-Muhtadi, S. Chetan, R. Campbell, and M.D. Mickunas, "MiddleWhere: a middleware for location-awareness in ubiquitous computing applications", in Proc. of Int. Middleware Conference, Middleware 2004, LNCS, vol. 3231, pp. 397-416.

[12] E. Mok, G. Retscher, and L. Xia, "Investigation of seamless indoor and outdoor positioning integrating WiFi and GNSS", in XXIII Int. FIG 2006 Congress, Munich, Germany, Oct 2006.

[13] R. Hansen, R. Wind, C.S. Jensen, and B. Thomsen, "Seamless indoor/outdoor positioning handover for location-based services in Streamspin", in Proc. of $10^{\text {th }}$ Int. Conf. on Mobile Data Management, MDM 2009, pp. 267-272, Taipei, Taiwan, May 2009

[14] A. Coronato and M. Esposito, "Towards an implementation of Smart Hospital: a localization system for mobile users and devices", in Proc. of $6^{\text {th }}$ IEEE Int. Conf. on Pervasive Computing and Communications, PerCom 2008, pp. 715-719, Hong Kong, Mar 2008.

[15] G. Vanderhulst, K. Luyten, and K. Coninx, "ReWiRe: creating interactive pervasive systems that cope with changing environments by rewiring", in Proc. of $4^{\text {th }}$ IET Int. Conf. on Intelligent Environments, IE'08, pp.1-8, Seattle, WA, USA, Jul 2008.

[16] K. Hinckley, J. Pierce, M. Sinclair, and E. Horovitz, "Sensing techniques for mobile interaction", in Proc. of $13^{\text {th }}$ Ann. ACM Symposium on User Interface Software and Technology, UIST 2000, pp.91-100, San Diego, CA, USA, Nov 2000.

[17] K. Leydon, "Sensing the position and orientation of hand-held objects: an overview of techniques", Technical report, University of Limerick, Dec 2001.

[18] Sonitor, High definition ultrasound indoor positioning systems, http://www.sonitor.com

[19] N. Franklin and B. Tversky, "Searching imagined environments", Journal of Experimental Psychology: General, vol. 119, pp.63-76, 1990.

[20] P. Aksenov, K. Luyten, and K. Coninx, "Reasoning over spatial relations for context-aware distributed user interfaces", in Proc. of $5^{\text {th }}$ Int. Workshop on Modelling and Reasoning in Context, MRC 2008, Delft, The Netherlands, Jun 2008, pp.37-50.

[21] M. Massink and G. Faconti, "A reference framework for continuous interaction", Universal Access in the Information Society, vol. 1, no. 4, pp. 237-251, 2002. 